\title{
SOME TOPICS ON EQUILIBRIA $\left({ }^{1}\right)$
}

\author{
BY \\ EZIO MARCHI
}

\begin{abstract}
In the present paper we introduce a proof for the existence of equilibrium points of a certain nonbilinear problem by using the Knaster-Kuratowski-Mazurkiewicz theorem, which turns out to be somewhat efficient for studies related to $n$-person games. As an application of this result, by embedding an n-person game in the "cooperative" set of action the existence of an equilibrium point in the strict noncooperative case and more general cases is obtained.
\end{abstract}

I. Introduction. Since the introduction of equilibrium point in noncooperative $n$-person games by Nash [10], [11], there have been many applications and some extensions in mathematical economics models of this very important concept. However, only recently has there appeared to be some interest in the outstanding problem of computing equilibria. There exists some general information about the structure of the equilibrium points set for an $n$-person game. See for example H. Chin, T. Parthasarathy and T. E. S. Raghavan [1]. However, in the case of two-person games, some interesting results by Vorobev [17], Kuhn [4] and Kreps [3] have been found.

In the present paper we introduce a proof for the existence of equilibrium points of a certain nonbilinear problem by using the Knaster-KuratowskiMazurkiewicz theorem, which turns out to be somewhat efficient for studies related to $n$-person games.

Indeed, as an application of this result, by embedding an $n$-person game in the "cooperative" set of actions the existence of an equilibrium point in the strict noncooperative case and more general cases is obtained.

Even though the new existence proof of equilibrium points is not by itself constructive, it holds to a computationally suitable way for the set of equilibrium points. In some specific cases, as for example, two-person games, one derives that such a set is the union of convex polyhedra. The extreme points of such polyhedra are computed by a similar technique as the important one developed by Kuhn [4].

Received by the editors February 13, 1974 and, in revised form, December 30, 1974. AMS (MOS) subject classifications (1970). Primary 90D10. Canada.

( ${ }^{1}$ )This research was supported by a grant from the National Research Council of 
Further, we introduce a section about transformation properties of equilibria.

Finally, we also extend von Neumann's result [16] for an $n$-person game with rational payoff functions.

We would like to point out that the same basic result allows one to study extensions of equilibrium point concepts as those $E$-points introduced by the author [6], [7], [9], which we will present in a separate report.

II. Basic results. Let $I$ be a nonempty finite set and $\tilde{I}$ be the set of all probability distribution defined on $I$, that is

$$
\tilde{I}=\left\{x \in R^{|I|}: x(i) \geqslant 0, \sum_{i \in I} x(i)=1, i \in I\right\},
$$

where $|I|$ indicates the number of elements in $I$ and $R$ is the set of real numbers. $x(i)$ is the $i$ th component of the vector $x \in R^{|I|}$. Given an $x \in \widetilde{I}$ let $S(x) \subset I$ be the support of $z$, that is, the set of $j \in I$ such that $z(j)>0$. Consider a continuous real function $G: I \times I \times \widetilde{I} \rightarrow R$. For any continuous transformation $p: \widetilde{I} \rightarrow \widetilde{I}$ we extend the function $G$ in a natural way by means of

$$
G(x, i, z)=\sum_{j \in I} p(x)(j) G(j, i, z) .
$$

We now have the following basic result:

THEOREM 1. If for each $z \in \tilde{I}$ there is an $i \in S(z)$ such that for any $j \in$ $S(p(z)), G(j, i, z) \geqslant 0$, then there is $a \bar{z} \in \tilde{I}$ such that $G(\bar{z}, i, \bar{z}) \geqslant 0$ for each $i \in I$.

Proof. For a given $i \in I$, define the set

$$
I(i)=\{z \in \tilde{I}: G(j, i, z) \geqslant 0 \text { for each } j \in S(p(z))\} .
$$

We want to see that such a set is closed. Consider a convergent sequence $z_{n} \rightarrow$ $z$ such that for each $n, z_{n} \in I(i)$. We note that there is an $n_{0}$ such that for all $n \geqslant n_{0}, S\left(p\left(z_{n}\right)\right) \supset S(p(z))$ since the transformation $p$ is continuous. Since $z_{n} \in I(i)$, then for each $j \in S\left(p\left(z_{n}\right)\right), G\left(j, i, z_{n}\right) \geqslant 0$. In particular, the above is true for each $j \in S(p(z))$. By the continuity of $G$, we have that for each $j \in$ $S(p(z)), G(j, i, z) \geqslant 0$. Therefore, $z \in I(i)$.

For any nonempty subset $J \subset I$, we indicate by $\widetilde{J}$ the natural embedding in $\tilde{I}$ of the corresponding probability distributions. Hence, by the condition imposed in the theorem, for any $z$ with $S(z)=J$ there is an $i \in J$ such that $z \in I(i)$. Therefore, for any nonempty subset $J \subset I: \widetilde{J} \subset \bigcup_{i \in J} I(i)$.

Consequently, the Knaster-Kuratowski-Mazurkiewicz theorem guarantees the nonemptiness of the intersection set $E=\bigcap_{i \in I} I(i)$. 
Any point $\bar{z} \in E$ satisfies $G(\bar{z}, i, \bar{z}) \geqslant 0$ for each $i \in I$. Q.E.D.

This result is too general for our purpose in this paper. We now will present an application of the above result which is more suitable for further studies concerning the existence of equilibria in game theory.

Consider a family of continuous real functions $f_{i}: \widetilde{I} \rightarrow R$ with $i \in I$ and define $F(i, z)=f_{i}(z)$. Then, the natural extension of $F$ to $\widetilde{I}$ is given by

$$
F(x, y)=\sum_{i \in I} p(x)(i) f_{i}(y)
$$

By applying Theorem 1, we obtain the next result:

THEOREM 2. If for each $z \in \tilde{I}: S(p(z)) \subset S(z)$, then there is $a \bar{z} \in \widetilde{I}$ such that $F(\bar{z}, \bar{z}) \geqslant F(i, \bar{z})$ for each $i \in I$.

Proof. Define $G(j, i, z)=F(j, z)-F(i, z)$. For any $z \in \tilde{I}$, let $\bar{i} \in S(p(z))$ be any $i \in S(p(z))$ such that $F(i, z)$ is a minimum with respect to the variable $i \in S(p(z))$. This obviously exists. Therefore, for each $j \in S(p(z)), F(j, z) \geqslant$ $F(\bar{i}, z)$ or equivalently $G(j, \bar{i}, z) \geqslant 0$. But $\bar{i} \in S(p(z)) \subset S(z)$. By the previous theorem, there exists a $\bar{z} \in \widetilde{I}$ such that $G(\bar{z}, i, \bar{z}) \geqslant 0$ for each $i \in I$. Such a point satisfies $F(\bar{z}, \bar{z}) \geqslant F(i, \bar{z})$ for each $i \in \tilde{I}$. Q.E.D.

A point satisfying Theorem 2 will be called an equilibrium point for $F$. One can characterize an equilibrium point in a somewhat slightly different way which will be useful in the subsequent parts. As an immediate consequence of Theorem 2, we have

COROllaRY 3. A point $\bar{z} \in \widetilde{I}$ is an equilibrium point for $F$ if and only if for each $j \in S(p(\bar{z})) F(j, \bar{z}) \geqslant F(i, \bar{z})$ for each $i \in I$.

Having the existence of an equilibrium point for $F$, we now want to see that any transformation $p$ having the condition established in Theorem 2 is onto.

LEMMA 4. For any continuous transformation $p: \widetilde{I} \rightarrow \widetilde{I}$, if for each $z \in$ $\widetilde{I}, S(p(z)) \subset S(z)$, then $p(\widetilde{I}) \supset \widetilde{I}$.

Proof. Clearly, since $\tilde{I}$ is convex, $p$ is homotopic to the identity map id. Indeed, $H(x, t)=t p(x)+(1-t) x$ is an homotopy bridge between $p$ and id.

Suppose that $p(\widetilde{I})$ is not $\widetilde{I}$; then there is a point $\bar{x} \in \tilde{I}$ which does not belong to the closed set $p(\widetilde{I})$. Therefore, for some $\epsilon>0$ all the points $x \in \widetilde{I}$ such that $|x-\bar{x}|<\epsilon, x \notin p(\widetilde{I})$. Let $\partial \widetilde{I}$ be the relative boundary of $\widetilde{I}$ and consider the continuous function $g: \widetilde{I}-p(\widetilde{I}) \rightarrow \partial \widetilde{I}$ which maps $x$ to the projection $g(x)$ $\in \partial \tilde{I}$ on the ray from $\bar{x}$ to $x$. The continuous composition $\lambda(x)=g(p(x))$ maps $\widetilde{I}$ into $\partial \widetilde{I}$. Its restriction $\lambda \mid \partial \widetilde{I}$ is indeed $p \mid \partial \tilde{I}$. Therefore, it is well known that $p \mid \partial \widetilde{I}$ is homotopic to a constant function in $\partial \tilde{I}$ (see, for example, Nikaido 
$[12$, p. 75]). Consequently, since $S(p(z)) \subset S(z)$ for each $z \in \tilde{I}, p \mid \partial \tilde{I}$ is homotopic to the identity in $\partial \widetilde{I}$, which implies that the identity map is homotopic to a constant map in $\partial \tilde{I}$. The Brouwer Fixed-Point Theorem assures that this is absurd. Q.E.D.

Going back to equilibrium points, we will present next a simple fact about the set of such points for different functions related by an appropriate transformation.

Let $\Delta(J)$ be the set of vectors $x \in R^{|I|}$ such that $x(j)=\mu_{x}$ for each $j \in J$ and $\mu_{x}>x(i)$ for each $i \notin J$. Then if $P(I)$ indicates the family of all nonempty subsets of $I$, we have that $\{\Delta(J), J \in P(I)\}$ is a partition of $R^{|I|}$.

We say that a transformation $\Omega: R^{|I|} \rightarrow R^{|I|}$ is order-preserving if $\Omega(\Delta(J))$ $\subset \Delta(J)$ for each $J \in P(I)$.

In what follows, all the transformations $p$ considered are assumed to have the property indicated in Theorem 2.

Given $F(i, \cdot)$ define $F_{\Omega}$ as the composition $F_{\Omega}=\Omega \circ F$; then one immediately has

PROPOSITON 5. If $\Omega$ is order-preserving, then an equilibrium point for $F$ is an equilibrium point for $F_{\Omega}$.

Proof. Let $J_{\bar{z}}$ be that subset of $I$ such that $F(., \bar{z}) \in \Delta\left(J_{\bar{z}}\right)$. Since $\Omega$ is order-preserving, $F_{\Omega}(\ldots, \bar{z}) \in \Delta\left(J_{\bar{z}}\right)$ or equivalently for each $j \in J_{\bar{z}}$ and $i \notin J_{\bar{z}}$, $F_{\Omega}(j, \bar{z})>F_{\Omega}(i, \bar{z})$. But $S(p(\bar{z})) \subset J_{\bar{z}}$ since $\bar{z}$ is an equilibrium point for $F$. Therefore, for each $j \in S(p(\bar{z})), F_{\Omega}(j, \bar{z}) \geqslant F_{\Omega}(i, \bar{z})$ for each $i \in I$. Q.E.D.

It is clear that the set of equilibrium points for $F$ is the intersection of all $I(i): E=\bigcap_{i \in I} I(i)$. Now, introducing the sets

$$
M(J, i)=\{z \in \widetilde{I}: S(p(z))=J \text { and } F(j, z) \geqslant F(i, z) \text { for each } j \in J\}
$$

we have that $I(i)=\bigcup_{J \in P(I)} M(J, i)$ and consequently

$$
E=\bigcap_{i \in I} \bigcup_{J \in P(I)} M(J, i)=\bigcup_{J \in P(I)} \bigcap_{i \in I} M(J, i) .
$$

Clearly, the set in the right term is always included in $E$. For any $\bar{z} \in E$ let $J_{\bar{z}}=S(p(\bar{z}))$, then $\bar{z} \in \bigcap_{i \in I} M\left(J_{\bar{z}}, i\right)$. Thus we have the previous equality.

The expression (1) characterizes the set of equilibria for $F$.

III. Properties in the bilinear case. Having the previous results, we now would like to study some properties regarding equilibria in the case in which the function $F$ is bilinear. Even though this case is quite particular, it appears to be quite wide reaching when applied to games. It incorporates general two-person games and a large variety of $n$-person games. 
In such an instance, it is possible to present some relevant properties and to have a technique for computational purposes. It is possible to compute all the extreme equilibria and therefore all the equilibrium points. Such a technique has many similarities with the very important method developed by Kuhn [4] for characterizing extreme optimal strategies in zero-sum two-person games.

Here in this section we consider that the function $F$ is given as

$$
F(x, y)=\sum_{i \in I} \sum_{j \in I} a_{i j} x(i) y(j)
$$

and the transformation $p$ is the identity. Therefore, in this case all the sets $M(J, i)$ become convex and the set of equilibrium points $E$ is a union of convex sets. We would like to remark that the sets $M(J, i)$, in general, are not closed.

Moreover, such sets are convex polyhedra. Consider the matrix $B^{i}=\left\{b_{j k}^{i}\right\}$ with $j, k \in J$ defined by $b_{j k}^{i}=a_{i k}-a_{j k}$. Then, it is clear that

$$
M(J, i)=K_{i}(J) \cap \widetilde{J}^{\circ}
$$

where $\widetilde{J}^{\circ}$ stands for the relative interior of $\widetilde{J}$ and

$$
K_{i}(J)=\left\{x \in \widetilde{J}: B^{i} x \leqslant 0\right\}
$$

Here $B^{i} x$ indicates the usual matrix multiplication.

Now, alternately the set $K_{i}(J)$ can be described by means of the set

$$
L_{i}(J)=\left\{(x, \lambda) \in \widetilde{J} \times R: B^{i} x-\lambda \leqslant 0 \text { and } \lambda \leqslant 0\right\} .
$$

Indeed, if $\pi_{J}$ is the projection on $\widetilde{J}$, we immediately have

$$
K_{i}(J)=\pi_{J} L_{i}(J)
$$

The points in $M(J, i)$, if any, can be obtained as a combination of its extreme points which might not belong to it. Thus, it is important to characterize the extreme points.

Write the linear system of inequalities

$$
\begin{aligned}
\sum_{k} b_{j k}^{l} x(k)-\lambda & \leqslant 0, \quad j \in J, \\
\sum_{k} x(k) & =1, \quad \lambda \leqslant 0,
\end{aligned}
$$

for $L_{i}(J)$.

For any given $m \times n$ matrix $A$, we write $\bar{A}$ as the $(m+1) \times(n+1)$ matrix having the entries $\bar{a}_{i j}=a_{i j}$ if $i \leqslant m$ and $j \leqslant n, \bar{a}_{m+1 j}=1$ for $j \leqslant n, \bar{a}_{i n+1}=$ -1 for $i \leqslant m$ and $\bar{a}_{m+1 n+1}=0$. Similarly $A^{\wedge}$ is the $(m+1) \times n$ matrix with entries $\hat{a}_{i j}=a_{i j}$ for $i \leqslant m$ and $j \leqslant n, \hat{a}_{m+1 j}=0$ if $j \leqslant m-1$ and $\hat{a}_{m+1 n}=1$. 
With these notations, we can express the above linear system of inequalities as

$$
\left(\bar{B}^{\wedge}\right)^{i}\left(\begin{array}{l}
x \\
\lambda
\end{array}\right) \leqslant v_{\bar{j}+1}
$$

where $v_{\bar{j}+1}=(0, \ldots, 1,0)$ is a $j+2$ vector with 1 in the $\bar{j}+1=(|J|+1)$ th position. We also use $u_{\bar{j}+1}=(0, \ldots, 1)$ as the unit $\bar{j}+1$ vector.

THEOREM 6. Let $\bar{x}, \bar{\lambda}$ be an extreme point of $L_{i}(J)$. If:

(a) $\bar{\lambda}<0$ there exists a $|S(\bar{x})| \times|S(\bar{x})|$ submatrix $Q$ of $B^{i}$ such that the rank $r(\bar{Q})$ of $\bar{Q}$ is $|S(\bar{x})|+1$ and $(\bar{x}, \bar{\lambda})$ is the solution of $\bar{Q}\left(\begin{array}{l}x \\ \lambda\end{array}\right)=u_{\bar{j}+1}$;

(b) $\bar{\lambda}=0$, there exists a $(|S(\bar{x})|-1) \times|S(\bar{x})|$ submatrix $Q$ of $B^{i}$ such that $r\left(\bar{Q}^{\wedge}\right)=|S(\bar{x})|+1$ and $(\bar{x}, 0)$ is the solution of $\bar{Q}^{\wedge}\left(\begin{array}{l}x \\ \lambda\end{array}\right)=v_{\bar{j}}$.

We skip the proof because it is essentially contained in Kuhn's paper.

As an immediate consequence of this theorem, we have that $(\bar{x}, \bar{\lambda})$ can be computed by Cramer's rule. Given a $q \times q$ matrix $Q$ we indicate by $Q_{i j}$ the $(i, j)$ th cofactor in $Q$, and $|Q|$ the determinant.

Corollary 7. If $\bar{x}, \bar{\lambda}$ is an extreme point of $L_{i}(J)$ and $Q$ the corresponding matrix specified in Theorem 6 , then

(a) if $\bar{\lambda}<0, x(k)=0$ for $k \in S(\bar{x})$ and

and

$$
\bar{x}(k)=\frac{\Sigma_{l} Q_{l k}}{|\bar{Q}|}=\frac{\Sigma_{l} Q_{l k}}{\Sigma_{l} \Sigma_{k} Q_{l k}}, \quad k \in S(\bar{x}),
$$

$$
\bar{\lambda}=\frac{|Q|}{|\bar{Q}|}=\frac{|Q|}{\Sigma_{l} \Sigma_{k} Q_{l k}} .
$$

(b) If $\bar{\lambda}<0: \bar{x}(k)=0, k \notin S(\bar{x})$ and

$$
\bar{x}(k)=\frac{Q_{k}^{*}}{\Sigma_{j} Q_{j}^{*}}
$$

where $Q_{k}^{*}$ is the $(q+1)$, kth cofactor in $Q^{*}$.

Since there are only a finite number of submatrices, it is possible to obtain effectively all the extreme points of the convex polyhedron $L_{i}(J)$.

We indicate by $E_{i}(J)$ the set of extreme points to $L_{i}(J)$. We have the following simple fact:

Proposition 8. The set $M(J, i)$ is nonempty if and only if $\bigcup_{x \in \pi_{J} E_{i}(J)} S(x)$ $=J$.

Proof. First the necessity. If $\bar{x} \in M(J, i)$ then $S(\bar{x})=J$ and for some $\bar{\lambda}$, $(\bar{x}, \bar{\lambda}) \in L_{i}(\nu)$. Thus, for some convex combination with $\mu_{k}>0$ :

$$
(\bar{x}, \bar{\lambda})=\left(\sum_{k} \mu_{k} x^{k}, \sum_{k} \mu_{k} \lambda^{k}\right)
$$


for some extreme points $\left(x^{k}, \lambda^{k}\right) \in E_{i}(J)$. But $S(\bar{x})=\bigcup_{k} S\left(x^{k}\right)$, and therefore $\bigcup_{\bar{x} \in \pi_{J} E_{i}(J)} S(x)=J$. For the sufficiency, let $\left(x^{k}, \lambda^{k}\right)$ be any family of extreme points such that $\bigcup_{k} S\left(x^{k}\right)=J$. Then any convex combination of them with $\mu_{k}$ $>0$ gives

$$
(x(\mu), \lambda(\mu))=\left(\sum_{k} \mu_{k} x^{k}, \sum_{k} \mu_{k} \lambda^{k}\right)
$$

which is in $L_{i}(J)$. Hence $x(\mu) \in K_{i}(J)$ and $S(x(\mu))=\bigcup_{k} S\left(x^{k}\right)$, implying that $x(\mu) \in M_{i}(J) \quad$ Q.E.D.

The intersection $\bigcap_{i \in I} M(J, i)$ will give all the equilibrium points for $F$ having support $J$. This may be characterized as a convex polyhedron by means of linear inequalities too.

It is interesting to note that although the set of equilibrium points $E$ is not, in general, convex, it can be entirely computed as convex combinations of extreme equilibrium points which in turn are extreme points to

$$
L(\widetilde{I})=\{x \in \widetilde{I}: A x \leqslant \lambda\} .
$$

THEOREM 9. If $\bar{x} \in E$ and $\left(\bar{x}, \lambda_{\bar{x}}\right)$ where $\lambda_{\bar{x}}=F(\bar{x}, \bar{x})$ is a strict convex combination of points $x^{k} \in L(\widetilde{I})$, then all $x^{k}$ are equilibrium points for $F$.

Proof. It is clear that if $\bar{x} \in E$ then $\left(\bar{x}, \lambda_{\bar{x}}\right) \in L(\widetilde{I})$. If it is a convex combination with $\mu_{k}>0$ :

$$
\left(\bar{x}, \lambda_{\bar{x}}\right)=\left(\sum_{k} \mu_{k} x^{k}, \sum_{k} \mu_{k} \lambda^{k}\right)
$$

where $\left(x^{k}, \lambda^{k}\right) \in L(\widetilde{I})$, then since $\left(\bar{x}, \lambda_{\bar{x}}\right)$ is an equilibrium point, we have $\Sigma_{k} \mu_{k} \lambda^{k} \geqslant \Sigma_{k} F\left(i, x^{k}\right)$ for all $i \in I$ and the strict equality for $i \in S(\bar{x})$. But $S(\bar{x})$ $=\bigcup_{k} S\left(x^{k}\right)$, and $\lambda^{k} \geqslant F\left(i, x^{k}\right)$ for all $i \in I$. In particular for $i \in S\left(x^{k}\right) \subset S(\bar{x}) \lambda^{k}$ $=F\left(x^{k}, x^{k}\right)=F\left(i, x^{k}\right)$, which implies that $\left(x^{k}, \lambda^{k}\right)$ is an equilibrium point. Q.E.D.

Consequently the set of extreme equilibrium points for $F: E \cap \pi_{I} L(\widetilde{I})$ is nonempty.

The points in $E \cap \pi_{I} L(\widetilde{I})$ are called extreme equilibrium points. By the last theorem, it is possible to compute all of them efficiently. This is the importance of such a result.

In order to reduce the computational procedure one might expect one extreme equilibrium point whose corresponding submatrix $\boldsymbol{P}$ is a principal minor. We hope to prove such a fact, among other results, in further studies.

IV. Equivalence classes. In this section, we will study a kind of equivalence relation for the set of bilinear functions $F$ considered in the previous section. This might be applied when $F$ also has the support inclusion property, but for reasons 
of simplicity we shall skip this case. All the functions in an equivalent class will have the same set of equilibrium points. Thus, the general problem is reduced only to a small family of functions. We will also mention some related combinatorial aspects.

Given a positive diagonal matrix $B$, define the transformation $\phi_{B}: \widetilde{I} \rightarrow \widetilde{I}$ as

$$
\phi_{B}(x)=\rho_{B}(x) B x \quad \text { where } \rho_{B}(x)=1 / \sum_{i} b_{i} x_{i}>0
$$

At once we have that $\rho$ satisfies

$$
\rho_{B}(a x)=\rho_{B}(x) / a, \quad \rho_{B_{1}}\left(B_{2} x\right)=\rho_{B_{1} B_{2}}(x)
$$

for any number $a$ and diagonal matrices $B_{1}$ and $B_{2}$.

Let $C$ be a matrix such that $C B=A$, where $F$ is the expectation of $A$. Then we have that $C \phi_{B}(x)-\rho_{B}(x) A x=0$ and from here for the expectation $F^{C}$ of $C$ we have

$$
F^{C}\left(j, \phi_{B}(\bar{z})\right)=\sum_{k} c_{j k}\left(\phi_{B}(\bar{z})\right)_{k}=\rho_{B}(x) \sum_{k} a_{j k} \bar{z}_{k}=\rho_{B}(x) F(j, \bar{z})
$$

for each $j \in I$ and $\bar{z} \in \tilde{I}$. If $\bar{z}$ happens to be an equilibrium point for $F$, then

$$
F^{C}\left(j, \phi_{B}(\bar{z})\right)=F(j, \bar{z}) \geqslant F(i, \bar{z})=F^{C}\left(i, \phi_{B}(\bar{z})\right)
$$

for all $j \in S(\bar{z})$ and $i \in I$, or in other words, $\phi_{B}(\bar{z})$ is an equilibrium point for $F^{C}$.

Thus, the set of equilibrium points for $F^{C}: E\left(A B^{-1}\right)=E(C)$ contains $\phi_{B}(E(A))$,

We also have for the composition $\phi_{B_{1}} \cdot \phi_{B_{2}}(x)=\phi_{B_{1} B_{2}}(x)$ and $\phi_{B}^{-1}=$ $\phi_{B-1}$. Furthermore $\phi_{B}(E(A))=E(C)$.

A further simple observation is that the matrix $A+I\left(k_{1}, \ldots, k_{\bar{n}}\right)$ (where the matrix $I\left(k_{1}, \ldots, k_{\bar{n}}\right)$ has the $i$ th column with all the entries $k_{i}$, and $\left.\bar{n}=|I|\right)$, has exactly the same set of equilibrium points as $A$. Indeed, it is immediate from the expectation expression:

$$
F^{k_{1}, \ldots, k_{\bar{i}}}(j, \bar{z})=F(j, \bar{z})+\sum_{i} k_{i} \bar{x}_{i}
$$

From this fact, one is allowed to study just payoff functions with all entries positive. The others will have a representative here with the same set of equilibria. Moreover, by the first consideration, given $A \geqslant 0$ with at least one positive entry in each column, if $b_{i i}=\Sigma_{k} a_{k i}>0$ then $A B$ is a stochastic matrix with respect to the columns and therefore all the remaining representatives are the stochastic matrices. 
Let $S$ be the set of all such stochastic matrices and consider the transformation

$$
\left[A+I\left(k_{1}, \ldots, k_{n}\right)\right] B^{-1}\left(k_{1}, \ldots, k_{n}\right)=C, A+I\left(k_{1}, \ldots, k_{n}\right) \geqslant 0
$$

for $A \in S$, with at least one positive entry in each column. Therefore, the matrix $B\left(k_{1}, \ldots, k_{n}\right)$ has entries $b_{j}\left(k_{1}, \ldots, k_{n}\right)=1+n k_{j}>0$.

It is clear that $C \in S$ and that

$$
E(C)=\phi_{B}(E(A))=\phi_{B}\left(E\left(A+I\left(k_{1}, \ldots, k_{n}\right)\right)\right) .
$$

It is interesting to note that the matrix $C$ can be obtained by inverting the operations. Indeed, let

$$
\bar{k}_{i}=-k_{i} /\left(1+n k_{i}\right)
$$

then, we have that $B\left(\bar{k}_{1}, \ldots, \bar{k}_{n}\right)$ is the inverse of $B\left(k_{1}, \ldots, k_{n}\right)$, since

$$
\begin{aligned}
0<b_{j}^{-1}\left(\bar{k}_{1}, \ldots, \bar{k}_{n}\right) & =1 /\left(1+n \bar{k}_{j}\right) \\
& =\left(1+n k_{j}\right) /\left(1+n k_{j}-n k_{j}\right) \\
& =1+n k_{j}=b_{j}\left(k_{1}, \ldots, k_{n}\right),
\end{aligned}
$$

and therefore

$$
\begin{aligned}
C+ & I\left(\bar{k}_{1}, \ldots, \bar{k}_{n}\right)=\left[A+I\left(k_{1}, \ldots, k_{n}\right)\right] B^{-1}\left(k_{1}, \ldots, k_{n}\right)+I\left(\bar{k}_{1}, \ldots, \bar{k}_{n}\right) \\
= & A B^{-1}\left(k_{1}, \ldots, k_{n}\right)+I\left(k_{1}, \ldots, k_{n}\right) B^{-1}\left(k_{1}, \ldots, k_{n}\right)+I\left(\bar{k}_{1}, \ldots, \bar{k}_{n}\right) \\
= & A B^{-1}\left(k_{1}, \ldots, k_{n}\right)+I\left(k_{1}, \ldots, k_{n}\right) B^{-1}\left(k_{1}, \ldots, k_{n}\right) \\
& -I\left(k_{1}, \ldots, k_{n}\right) B^{-1}\left(k_{1}, \ldots, k_{n}\right) \\
= & A B^{-1}\left(k_{1}, \ldots, k_{n}\right)=A B\left(\bar{k}_{1}, \ldots, \bar{k}_{n}\right) \geqslant 0
\end{aligned}
$$

with at least one positive entry in each column. Equivalently

$$
\left[C+I\left(\bar{k}_{1}, \ldots, \bar{k}_{n}\right)\right] B^{-1}\left(\bar{k}_{1}, \ldots, \bar{k}_{n}\right)=A .
$$

Thus,

$$
E(C)=E\left(A B^{-1}\left(k_{1}, \ldots, k_{n}\right)\right)=\phi_{B}(E(A))
$$

From here, we can define an equivalent relation in $S$ as $A_{2} R A_{1}$ if and only if there is a vector $\left(k_{1}, \ldots, k_{n}\right)$ such that

$$
A_{2}=\left[A_{1}+I\left(k_{1}, \ldots, k_{n}\right)\right] B^{-1}\left(k_{1}, \ldots, k_{n}\right)
$$


where $A_{1}+I\left(k_{1}, \ldots, k_{n}\right) \geqslant 0$ has at least one positive entry in each column.

It is simple to verify that $R$ is an equivalent relation.

As a consequence we have that the computation of equilibrium points is reduced to the equivalence classes. For any two stochastic matrices in the same equivalence class the set of equilibria are in a one-to-one correspondence given above.

It is worth mentioning that the existence of an equilibrium point for $F$ is equivalent to the very combinatorial fact that $\bigcup_{J \in P(I)} \Delta_{F}(J)$ is nonempty for any stochastic matrix, where here $\Delta_{F}(J)=\{z: S(z)=J$ and $F(z, z) \in \Delta(J)\}$.

V. Equilibrium points in $n$-person games. Having studied some properties in the previous section which allow the complete computation of all equilibria in the bilinear case, we will now go on to present some interesting applications to $n$-person games.

As a first simple fact, consider the case where $F(x, y)=E(x, y)-E(y, x)$ and $E$ is the expectation of a given matrix $A$. Then, immediately from Theorem 2 we have

COROLLARY 10. There exists a point $\bar{x}$ such that $E(\bar{x}, i) \geqslant E(i, \bar{x})$ for every $i \in I$.

Proof. Indeed, let $\bar{x}$ be an equlibrium point for $F$ :

$$
F(\bar{x}, \bar{x}) \geqslant F(i, \bar{x})=E(i, \bar{x})-E(\bar{x}, i)
$$

for each $i \in I$. But $F(\bar{x}, \bar{x})=0$ since $F$ is skew-symmetric. Q.E.D.

Even this result implies the minimax theorem, which is precisely the method adopted by Nikaido-Isoda [13].

Now, we study $n$-person games. Let $\Gamma=\left\{\Sigma_{i}, A_{i} ; i \in N\right\}$ be a finite $n$-person game in normal form. Let $\Sigma=X_{i \in N} \Sigma_{i}$ and consider continuous functions $p: \widetilde{\Sigma} \rightarrow \widetilde{\Sigma}$ and $q^{i}: \widetilde{\Sigma}_{j \neq i} \rightarrow \widetilde{\Sigma}_{j}$ for each $i \in N$, where $p$ is such that $S(p(z)) \subset$ $S(z)$ for each $z \in \widetilde{\Sigma}$. Therefore, we define

$$
\begin{aligned}
& f_{\sigma_{i}}^{i}(z)=\sum_{\tau_{1}, \ldots, \tau_{i-1}, \tau_{i+1}, \ldots, \tau_{n}} A_{i}\left(\tau_{1}, \ldots, \tau_{i-1}, \sigma_{i}, \tau_{i+1}, \ldots, \tau_{n}\right) \\
& \quad \cdot q^{i}(z)\left(\tau_{1}, \ldots, \tau_{i-1}, \tau_{i+1}, \ldots, \tau_{n}\right) \\
& =E_{i}\left(\sigma_{i}, q^{i}(z)\right), \begin{array}{l}
f_{\sigma}(z)=\sum_{i \in N} f_{\sigma_{i}}^{i}(z)
\end{array}
\end{aligned}
$$

for $\sigma=\left(\sigma_{1}, \ldots, \sigma_{n}\right) \in \Sigma$ and $z \in \widetilde{\Sigma}$ and for $x, y \in \widetilde{\Sigma}$ : 


$$
\begin{aligned}
F(x, y) & =\sum_{\sigma_{1}, \ldots, \sigma_{n}} p(x)\left(\sigma_{1}, \ldots, \sigma_{n}\right) f_{\sigma}(y) \\
& =\sum_{i \in N} \sum_{\sigma_{1}, \ldots, \sigma_{n}} p(x)\left(\sigma_{1}, \ldots, \sigma_{n}\right) f_{\sigma_{i}}^{i}(y) \\
& =\sum_{i \in N} \sum_{\sigma_{i}}\left(\sum_{\sigma_{1}, \ldots, \sigma_{i-1}, \sigma_{i+1}, \ldots, \sigma_{n}} p(x)\left(\sigma_{1}, \ldots, \sigma_{n}\right)\right) E_{i}\left(\sigma_{i}, q^{i}(y)\right) \\
& =\sum_{i \in N} E_{i}\left(\pi_{i}(p(x)), q^{i}(y)\right)
\end{aligned}
$$

where $\pi_{i}: \widetilde{\Sigma} \rightarrow \widetilde{\Sigma}_{i}$ is the natural projection giving the marginal probability.

By Theorem 2 , there exists a $\bar{z} \in \widetilde{\Sigma}$ such that

$$
\sum_{i \in N} E_{i}\left(\pi_{i}(p(\bar{z})), q^{i}(z)\right) \geqslant \sum_{i \in N} E_{i}\left(\pi_{i}(p(z)), q^{i}(\bar{z})\right)
$$

for each $z \in \widetilde{\Sigma}$. Let

$$
\lambda\left(z_{1}, \ldots, z_{n}\right)=\bigcap_{i \in N} \pi_{i}^{-1}\left(z_{i}\right)
$$

for $z_{i} \in \widetilde{\Sigma}_{i}$ which can be seen to be a convex polyhedron [8]. We call it the fiber. Intuitively speaking this is the set of all cooperative actions among the players generated by the individual mixed strategies $z_{i} \in \widetilde{\Sigma}_{i}$. Consider further the set $p^{-1}\left(\lambda\left(z_{1}, \ldots, z_{n}\right)\right)$. Since $p$ is onto, $\pi_{i} p: \widetilde{\Sigma} \rightarrow \widetilde{\Sigma}_{i}$ is also onto.

Taking any $z_{i} \in \widetilde{\Sigma}_{i}$ and any

$$
z \in p^{-1}\left(\lambda\left(\pi_{1}(\bar{z}), \ldots, \pi_{i-1}(\bar{z}), z_{i}, \pi_{i+1}(\bar{z}), \ldots, \pi_{n}(\bar{z})\right)\right)
$$

inequality (*) becomes

$$
E_{i}\left(\pi_{i}(p(\bar{z})), q^{i}(\bar{z})\right) \geqslant E_{i}\left(\pi_{i}(p(z)), q^{i}(\bar{z})\right)
$$

Thus, $\bar{z} \in \widetilde{\Sigma}$ is such that for each $i \in N$, each $z_{i} \in \widetilde{\Sigma}_{i}$ and each

$$
\begin{gathered}
z \in p^{-1}\left(\lambda\left(\pi_{1}(\bar{z}), \ldots, \pi_{i-1}(\bar{z}), z_{i}, \pi_{i+1}(\bar{z}), \ldots, \pi_{n}(\bar{z})\right)\right), \\
E_{i}\left(\pi_{i}(p(\bar{z})), q^{i}(\bar{z})\right) \geqslant E_{i}\left(\pi_{i}(p(z)), q^{i}(\bar{z})\right) .
\end{gathered}
$$

We call such a point a $(p, q)$-equilibrium point for the $n$-person game $\Gamma$. Therefore we have proved

THEOREM 11. For any finite n-person game $\Gamma$ and any continuous functions $q^{i}, p$ such that $S(p(z)) \subset S(z)$ for each $z \in \widetilde{\Sigma}$, there exists $a(p, q)$ equilibrium point.

The previous result which gives the existence of an equilibrium point in the nonmultilinear case is a generalization of Nash's classical result about equilibrium points [8] and [9]. 
Consider the case where

$$
\bar{q}^{i}(z)\left(\sigma_{1}, \ldots, \sigma_{i-1}, \sigma_{i+1}, \ldots, \sigma_{n}\right)=\prod_{j \neq i} \pi_{j}(z)\left(\sigma_{j}\right),
$$

for each $i \in N$, and $p$ is the identity mapping. Then, the projection point $\left(\pi_{1}(\bar{z}), \ldots, \pi_{n}(\bar{z})\right) \in X_{i \in N} \widetilde{\Sigma}_{i}$ of any (id, $\left.\bar{q}\right)$-equilibrium point $\bar{z}$, since the expectations $E_{i}$ are now independent of all points in the fiber, is indeed a Nash equilibrium point in the mixed extension $\widetilde{\Gamma}=\left\{\widetilde{\Sigma}_{i}, E_{i} ; i \in N\right\}$.

This takes into account only the strictly noncooperative actions.

Now, we would like to study the relationship between the set of (id, $\bar{q}$ )equilibrium points for $\Gamma$ and the set of Nash equilibrium points. We recall that the former set is

$$
E^{c}=\bigcup_{T \in P(\Sigma)} \bigcap_{\sigma \in \Sigma} M(T, \sigma)
$$

where the $M$ 's set are considered with respect to the $F$ function. On the other hand, it is easy to see that the set of Nash equilibrium points is given by

$$
E^{n c}=\bigcup_{T \in Q(\Sigma)} \bigcap_{\sigma \in \Sigma} N(T, \sigma)
$$

where $\pi_{i}(T)=T_{i}$ is the projection of the set $T \subset \Sigma$ into $\Sigma_{i}, Q(\Sigma)$ is the family of all nonempty subsets $T=\mathrm{X}_{i \in N} \pi_{i}(T)$ in $\Sigma$ and $N(T, \sigma)$ is the set of all $z \in$ $\times_{i \in N} \widetilde{\Sigma}_{i}$ such that $S\left(z_{i}\right)=T_{i}$ and for each $i \in N$ :

(2) $E_{i}\left(z_{1}, \ldots, z_{i-1}, \tau_{i}, z_{i+1}, \ldots, z_{n}\right) \geqslant E_{i}\left(z_{1}, \ldots, z_{i-1}, \sigma_{i}, z_{i+1}, \ldots, z_{n}\right)$

for each $\tau_{i} \in T_{i}$.

Proposition 12. For any finite game $\Gamma$, given $p=$ id and $\bar{p}$, then $E^{c}=$ $\lambda\left(E^{n c}\right)$.

Proof. Given a $T \in Q(\bar{z})$, let $R(T)$ be the family of subsets $U \subset T$ such that $\pi_{i}(U)=\pi_{i}(T)=T_{i}$ for each $i \in N$. Then, we can write

$$
E^{c}=\bigcup_{T \in Q(z)} \bigcup_{U \in R(T)} \bigcap_{\sigma \in \Sigma} M(U, \sigma) .
$$

We first want to see that

$$
\bigcup_{U \in R(T)} \bigcap_{\sigma \in \Sigma} M(U, \sigma)=\lambda\left(\bigcap_{\sigma \in \Sigma} N(T, \sigma)\right) .
$$

Let $z$ be a point in the first set, then $S(z)=U$ for some $U \in R(T)$. Therefore for $z_{i}=\pi_{i}(z)$, we have $S\left(\pi_{i}(z)\right)=T_{i}=\pi_{i}(T)=\pi_{i}(U)$. Let $\tau_{i} \in T_{i}$ for some $i \in N$, then there is some $\bar{\tau} \in U$ such that $\bar{\tau}_{i}=\tau_{i}$. Since $z \in \bigcap_{\sigma \in \Sigma} M(U, \sigma)$ we have that 


$$
\begin{aligned}
\sum_{i \in N} E_{i}\left(z_{1}, \ldots, z_{i-1}, \tau_{i}, z_{i+1}, \ldots, z_{n}\right) & \\
& \geqslant \sum_{i \in N} E_{i}\left(z_{1}, \ldots, z_{i-1}, \sigma_{i}, z_{i+1}, \ldots, z_{n}\right)
\end{aligned}
$$

for each $\tau \in U$ and $\sigma \in \Sigma$. In particular for $\bar{\tau} \in U$ and $\bar{\sigma}=\left(\bar{\tau}_{1}, \ldots, \bar{\tau}_{i-1}, \sigma_{i}\right.$, $\bar{\tau}_{i+1}, \ldots, \bar{\tau}_{n}$ ), from here, we obtain (2) for each $i \in N, \tau_{i} \in T_{i}$ and $\sigma_{i} \in \Sigma_{i}$. This implies that $\left(z_{1}, \ldots, z_{n}\right) \in \bigcap_{\sigma \in \Sigma} N(T, \sigma)$.

Reciprocally, given $\left(z_{1}, \ldots, z_{n}\right)$ in $\bigcap_{\sigma \in \Sigma} N(T, \sigma)$, consider any $z \in$ $\lambda\left(z_{1}, \ldots, z_{n}\right)$ with $S(z)=U \in R(T)$. Given $\tau \in U$ and any $\sigma \in \Sigma$, since $\left(z_{1}, \ldots, z_{n}\right) \in N(T, \sigma)$ and $\tau_{i} \in S\left(z_{i}\right)=T_{i}$ we have (2), and adding them:

$$
\begin{aligned}
\sum_{i \in N} E_{i}\left(z_{1}, \ldots,\right. & \left.z_{i-1}, \tau_{i}, z_{i+1}, \ldots, z_{n}\right) \\
& \geqslant \sum_{i \in N} E_{i}\left(z_{1}, \ldots, z_{i-1}, \sigma_{i}, z_{i+1}, \ldots, z_{n}\right),
\end{aligned}
$$

or equivalently, $z \in M(U, \sigma)$.

Therefore

$$
E^{c}=\bigcup_{T \in Q(\Sigma)} \lambda\left(\bigcap_{\sigma \in \Sigma} N(T, \sigma)\right)=\lambda\left(\bigcup_{T \in Q(\Sigma)} \bigcap_{\sigma \in \Sigma} N(T, \sigma)\right)=E^{n c}
$$

which is the desired result. Q.E.D.

It is worth observing that the set of equilibrium points in $n$-person games has a particular shape.

Given a set $K \subset \times_{i \in N} K_{i}$ where $K_{i}$ is a convex set in an euclidean space, we say that $K$ is multiconvex if for any

$$
\begin{aligned}
& x=\left(x_{1}, \ldots, x_{j-1}, x_{j}, x_{j+1}, \ldots, x_{n}\right), \\
& y=\left(x_{1}, \ldots, x_{j-1}, y_{j}, x_{j+1}, \ldots, x_{n}\right)
\end{aligned}
$$

in $K$ any convex combination $\mu x+(1-\mu) y \in K$. In the case that $|N|=1$ this notion becomes the usual concept of convexity. Thus,

It is clear at once that the set $\bigcap_{\sigma \in \Sigma} N(T, \sigma) \subset \times_{i \in N} \widetilde{\Sigma}_{i}$ is multiconvex.

COROLlaRY 13. The set of Nash equilibrium points of any finite n-person game is the union of multiconvex sets.

However, there is a particular case where the shape of the equilibrium points set is simpler, namely, when the expectations $E_{i}$ are linear functions in the variables $\left(z_{1}, \ldots, z_{i-1}, z_{i+1}, \ldots, z_{n}\right) \in X_{j \neq i} \widetilde{\Sigma}_{j}$. Consider any permutation $\theta: N \rightarrow N$ without fixed points. In the case that the payoff functions are $A_{i}\left(\sigma_{1}, \ldots, \sigma_{n}\right)=A_{i}\left(\sigma_{i}, \sigma_{\theta(i)}\right)$, the expectations will be $E_{i}\left(z_{i}, z_{\theta(i)}\right)$. Therefore 
all the sets $N(T, \sigma)$ become convex. Similarly the function $F$ is bilinear and consequently both equilibrium sets $E^{c}$ and $E^{n c}$ are unions of convex sets. This is certainly the case for two-person games. Furthermore, all the extreme points of $E^{c}$ can be effectively computed by the methods indicated in §III. Such extreme points will without any doubt be related to the extremes of $E^{n c}$. Some other examples can also be considered.

VI. Equilibrium points for rational $n$-person games. Having presented in the previous section the application of our basic result to $n$-person games, we now will show that it is possible to get more general results in the same direction. Indeed, we will obtain the existence of general equilibrium points for games with rational payoff functions. This was recently done by the author [9] by using Kakutani's fixed point theorem, thus obtaining a generalization of von Neumann's result [16].

Here we will use a further approach based on the results in §II.

In this section, let $\Gamma=\left\{\Sigma_{i}, A_{i} ; i \in N\right\}$ be a finite $n$-person game where the payoff functions $A_{i}=B_{i} / C_{i}$ where $C_{i}>0$. For given $q^{i}, p$ as in the previous section, define

$$
g_{\sigma_{i}}^{i}(z)=E_{i}^{C}\left(\pi_{i}(p(z)), q^{i}(z)\right) E_{i}^{B}\left(\sigma_{i}, q^{i}(z)\right)-E_{i}^{B}\left(\pi_{i}(p(z)), q^{i}(z)\right) E_{i}^{C}\left(\sigma_{i}, q^{i}(z)\right)
$$

for $\sigma \in \Sigma$ and $z \in \widetilde{\Sigma}$, where $E_{i}^{B}$ and $E_{i}^{C}$ are the expectations of $B_{i}$ and $C_{i}$ respectively. From here,

$$
\begin{aligned}
G(x, y)= & \sum_{\sigma_{1}, \ldots, \sigma_{n}} p(x)\left(\sigma_{1}, \ldots, \sigma_{n}\right) g_{\sigma}(y) \\
= & \sum_{\sigma_{1}, \ldots, \sigma_{n}} p(x)\left(\sigma_{1}, \ldots, \sigma_{n}\right), \sum_{i \in N} g_{\sigma_{i}}^{i}(y) \\
= & \sum_{i \in N}\left[E_{i}^{C}\left(\pi_{i}(p(y)), q^{i}(y)\right) E_{i}^{B}\left(\pi_{i}(p(x)), q^{i}(y)\right)\right. \\
& \quad-E_{i}^{B}\left(\pi_{i}(p(y)), q^{i}(y)\right) E_{i}^{C}\left(\pi_{i}(p(x)), q^{i}(y)\right]
\end{aligned}
$$

for $x, y \in \widetilde{\Sigma}$. By Theorem 2, there exists a $\bar{z} \in \widetilde{\Sigma}$ such that $G(\bar{z}, \bar{z}) \geqslant G(z, \bar{z})$ for each $z \in \widetilde{\mathbf{\Sigma}}$. Replacing its value in terms of the expectation:

$$
\begin{aligned}
G(\bar{z}, \bar{z})=0 \geqslant \sum_{i \in N}\left[E_{i}^{C}\left(\pi_{i}(p(\bar{z})), q^{i}(\bar{z})\right) E_{i}^{B}\left(\pi_{i}(p(z)), q^{i}(\bar{z})\right)\right. \\
\left.-E_{i}^{B}\left(\pi_{i}(p(\bar{z})), q^{i}(\bar{z})\right) E_{i}^{C}\left(\pi_{i}(p(z)), q^{i}(\bar{z})\right)\right]
\end{aligned}
$$


or

$$
\begin{aligned}
\sum_{i \in N} E_{i}^{B}\left(\pi_{i}(p(\bar{z})),\right. & \left.q^{i}(\bar{z})\right) E_{i}^{C}\left(\pi_{i}(p(z)), q^{i}(\bar{z})\right) \\
& \geqslant \sum_{i \in N} E_{i}^{C}\left(\pi_{i}(p(\bar{z})), q^{i}(\bar{z})\right) E_{i}^{B}\left(\pi_{i}(p(z)), q^{i}(\bar{z})\right)
\end{aligned}
$$

for each $z \in \widetilde{\Sigma}$.

Taking any $z_{i} \in \widetilde{\Sigma}_{i}$ and any

$$
z \in p^{-1}\left(\lambda\left(\pi_{1}(\bar{z}), \ldots, \pi_{i-1}(\bar{z}), z_{i}, \pi_{i+1}(\bar{z}), \ldots, \pi_{n}(\bar{z})\right)\right)
$$

consecutively in the last inequality, we have that $\bar{z} \in \widetilde{\Sigma}$ is such that for each $i \in$ $N$, each $z_{i} \in \widetilde{\Sigma}_{i}$ and each

$$
\begin{aligned}
& z \in p^{-1}\left(\lambda\left(\pi_{1}(\bar{z}), \ldots, \pi_{i-1}(\bar{z}), z_{i}, \pi_{i+1}(\bar{z}), \ldots, \pi_{n}(\bar{z})\right)\right) \\
& E_{i}^{B}\left(\pi_{i}(p(\bar{z})), q^{i}(\bar{z})\right) E_{i}^{C}\left(\pi_{i}(p(z)), q^{i}(\bar{z})\right) \\
& \quad \geqslant E_{i}^{C}\left(\pi_{i}(p(\bar{z})), q^{i}(\bar{z})\right) E_{i}^{B}\left(\pi_{i}(p(z)), q^{i}(\bar{z})\right)
\end{aligned}
$$

or

$$
\frac{E_{i}^{B}\left(\pi_{i}(p(\bar{z})), q^{i}(\bar{z})\right)}{E_{i}^{C}\left(\pi_{i}(p(\bar{z})), q^{i}(\bar{z})\right)} \geqslant \frac{E_{i}^{B}\left(\pi_{i}(p(z)), q^{i}(\bar{z})\right)}{E_{i}^{C}\left(\pi_{i}(p(z)), q^{i}(\bar{z})\right)}
$$

since all $C_{i}$ are strictly positive.

We say that such a point is an $(p, q)$-equilibrium point for the rational $n$ person game $\Gamma$. We have proved

THEOREM 14. Any rational n-person game $\Gamma$ has a $(p, q)$-equilibrium point if $p, q^{i}$ are continuous and $S(p(z)) \subset S(z)$ for each $z \in \widetilde{\Sigma}$.

In the case when $p=$ id and $q^{i}=\bar{q}^{t}$ as in the last paragraph, then the projection $\left(\pi_{1}(\bar{z}), \ldots, \pi_{n}(\bar{z})\right) \in X_{i \in N} \widetilde{\Sigma}_{i}$ is an equilibrium point in mixed strategies.

We observe that if all the payoff functions $C_{i}$ are identically one, all the expectations $E_{i}^{C}$ are identically one also, and therefore Theorem 14 particularizes into the result given in Theorem 1.

Finally, we would like to point out that many results can be extended in the direction given in [6] and [7] for $E$-points and some more general concepts of stability. Furthermore, considering the results in [8] some natural extensions in the cooperative context can be developed.

\section{BIBLIOGRAPHY}

1. H. Chin, T. Parthasarathy and T. E. S. Raghavan, Structure of equilibria in $N$-person non-cooperative games, Internat. J. Game Theory 3 (1974), 1-19. MR 49 \#8672. 
2. S. Karlin, Mathematical methods and theory in games, programming and economics. Vol. I: Matrix games, programming, and mathematical economics, Addison-Wesley, Reading, Mass., 1959. MR 22 \#2496.

3. V. L. Kreps, Uniqueness of the equilibrium point in bimatrix games, Internat. J. Game Theory 3 (1974), 115-118.

4. H. W. Kuhn, An algorithm for equilibrium points in bimatrix games, Proc. Nat. Acad. Sci. U.S.A. 47 (1961), 1657-1662. MR 24 \#B2036.

5. E. Marchi, On the minimax theorem of the theory of games, Ann. Mat. Pura Appl. (4) 77 (1967), 207-282. MR 38 \#1914.

6. $\longrightarrow$ E-points of games, Proc. Nat. Acad. Sci. U.S.A. 57 (1967), 878-882. MR 35 \#7702.

7. Fundamentals of non-cooperative games, Econ. Res. Program, Princeton Univ. Res. Memo. No. 97, 1968, p. 315.

8. The natural vector bundle of the set of product probability, $\mathrm{Z}$. Wahrscheinlichkeitstheorie und Verw. Gebiete 23 (1972), 7-17. MR 47 \#4301. appear).

9. - Equilibrium points of rational n-person games, J. Math. Anal. Appl. (to

10. J. F. Nash, Equilibrium points in n-person games, Proc. Nat. Acad. Sci. U.S.A. 36 (1950), 48-49. MR 11, 192.

11. - Non-cooperative games, Ann of Math. (2) 54 (1951), 286-295. MR 13, 261.

12. H. Nikaidô, Convex structures and economic theory, Math. in Sci. and Engineering, vol. 51, Academic Press, New York and London, 1968. MR 43 \#2970.

13. $\mathrm{H}$. Nikaido and $\mathrm{K}$. Isoda, Note on non-cooperative convex games, Pacific J. Math. 5 (1955), 807-815. MR 17, 506.

14. L. S. Shapley and R. N. Snow, Basic solutions of discrete games, Contributions to the Theory of Games. Vol. I, Ann. of Math. Studies, no. 24 (H. W. Kuhn and A. W. Tucker, Editors), Princeton Univ. Press, Princeton, N. J., 1950, pp. 27-35. MR 12, 513.

15. L. S. Shapley, On balanced sets and cores, Naval Res. Logist. Quart. 14 (1967), $453-460$.

16. J. von Neumann, Über ein ökonomisches Gleichnungssystem und eine Verallgemeinerung des Brouwerschen Fixpunktsatzes, Ergebnisse eines Mathematischen Kolloquiums 8 (1937), 73-83.

17. N. N. Vorob'ev, Equilibrium points in bimatrix games, Teor. Verojatnost. i Primenen 3 (1958), 313-331 = Theor. Probability Appl. 3 (1958), 297-309. MR 20 \#6948. 80-87.

18. R. Wilson, Computing equilibria of n-person games, Siam J. Appl. Math. 21 (1971),

DEPARTAMENTO DE MATEMATICAS, UNIVERSIDAD DE SAN LUIS, SAN LUIS, ARGENTINA 